Revue d'histoire de l'Amérique française

REYUE D.HISTOIRE DE L'AMÉRIQUE FRANÇAISE

\title{
ANCTIL, Pierre, Ira ROBINSON et Gérard BOUCHARD, dir., Juifs et Canadiens français dans la société québécoise (Sillery, Septentrion, 2000), 200 p.
}

\section{Yolande Cohen}

Volume 56, numéro 3, hiver 2003

URI : https://id.erudit.org/iderudit/007620ar

DOI : https://doi.org/10.7202/007620ar

Aller au sommaire du numéro

Éditeur(s)

Institut d'histoire de l'Amérique française

ISSN

0035-2357 (imprimé)

1492-1383 (numérique)

Découvrir la revue

Citer ce compte rendu

Cohen, Y. (2003). Compte rendu de [ANCTIL, Pierre, Ira ROBINSON et Gérard BOUCHARD, dir., Juifs et Canadiens français dans la société québécoise (Sillery, Septentrion, 2000), 200 p.] Revue d'histoire de l'Amérique française, 56(3),

391-394. https://doi.org/10.7202/007620ar d'utilisation que vous pouvez consulter en ligne. 
ANCTIL, Pierre, Ira ROBINSON et Gérard BOUCHARD, dir., Juifs et Canadiens français dans la saciété québécoise (Sillery, Septentrion, 2000), 200 p.

Publier les actes d'un colloque relève toujours de la gageure, tant la collection de textes énoncés à un moment donné peut sembler disparate et peu cohérente dans un même ouvrage. Pourtant, l'on doit aux organisateurs du colloque le mérite d'avoir poursuivi avec cet ouvrage la réflexion amorcée durant le colloque. Organisé conjointement par l'Institut interuniversitaire de recherches sur les populations (IREP) et la Bibliothèque publique juive de Montréal, ce colloque tenu en mars 1999 se voulait une rencontre scientifique, avec, selon le mot des deux organisateurs de l'événement, Gérard Bouchard et Zipporah Shnay, un objectif social et culturel (p. 7). L'ouvrage présente donc les contributions d'historiens connus pour leurs travaux sur les Juifs et sur les Canadiens français, dont les directeurs du recueil, ainsi que celles de chercheurs et de personnalités de l'une ou l'autre communauté. Et même si un des objectifs du colloque était d'assurer «une réciprocité des interrogations et des réponses, afin de dresser un portrait dynamique et aussi complet que possible des relations judéo-québécoises» (p. 9), les contributions publiées témoignent de la difficulté de réaliser une telle entreprise. Car les stéréotypes ont la vie dure, de part et d'autre, comme on le voit encore aujourd'hui; mais du moins, cet effort de réflexion conjointe a-t-il le mérite d'exister.

Abordée d'emblée, la question de l'antisémitisme est celle qui soulève le plus de controverses. Si Gérard Bouchard reconnaît l'existence de différents épisodes d'un antisémitisme limité au Québec à certaines élites (de la part "des éléments plus conservateurs du clergé, des professions libérales et du milieu enseignant», p. 21) et à la période 1880-1940, il recommande aussi de montrer «les relations pacifiques, les actes de solidarité, les initiatives de rapprochement", avant 1840 par exemple (p. 21) et à partir de 1947. Plus encore, se penchant sur les causes de l'antisémitisme, il ajoute à la thèse de Pierre Anctil (les juifs porteurs de la modernité menaçant les valeurs traditionnelles), celle du «développement collectif remarquable (de la communauté juive) alors que les Canadiens français empruntaient un cheminement plus incertain ", ce qui aurait assuré aux Juifs une mobilité sociale ascendante aux côtés des anglophones. Ainsi l'auteur émet l'hypothèse que les «expressions d'antisémitisme canadien-français visaient moins le Juif lui-même que l'allié d'une classe dominante réprouvée... Le Juif [serait] une sorte de Canadien français inversé qui aurait renoncé à une partie de son identité pour sortir de sa condition de défavorisé» (p. 27). Il 
est étonnant de retrouver sous la plume du brillant historien des explications aussi sommaires et stéréotypées. Car s'il est une tautologie connue des chercheurs dans ce domaine, c'est bien celle qui fait de la minorité exclue la responsable de son oppression (le syndrome bien connu du Blame the victim). S'il évite de mentionner l'explication la plus usuelle de l'antisémitisme comme une manifestation d'un antijudaïsme par des élites catholiques, Bouchard aurait dû au moins soulever la question de savoir pourquoi les élites conservatrices canadiennes-françaises avaient identifié la petite élite de marchands juifs comme responsable de la modernisation qu'ils redoutaient tant? Ce qui lui aurait permis de souligner la forte propension de ces élites au corporatisme, qu'il connaît fort bien par ailleurs. Cette impasse sur cette forme de nationalisme canadien-français lui permet tout naturellement de s'interroger sur «la réticence des Juifs à l'endroit du nationalisme et du projet de souveraineté dont il est porteur» (p. 28).

Et nous revoilà en plein débat national, annoncé en introduction par le chercheur qui considère «le rapport à instaurer avec la communauté juive comme un test pour la nation québécoise en tant que projet d’intégration dans la diversité» (p. 14). Nous retrouvons ici les préoccupations politiques de Gérard Bouchard, dont l'ambition est de proposer une mise en forme de la nation québécoise, en proposant une alternative au multiculturalisme canadien. Si ce projet est fort louable et entièrement légitime, il aurait fallu établir les distinctions qui s'imposent entre le vieux nationalisme, corporatiste et antisémite des années 1920 et 1930, et le projet de souveraineté que Bouchard entrevoit, ouvert et intégrateur des différences.

Il faudrait également redonner leur place à chacun des acteurs d'un tel projet politique. À commencer par l'usage du mot communautés, qui suggère une équivalence entre les places occupées par Juifs et Canadiens français dans la société québécoise. Car comment parler des rapports entre minorité (juive) et majorité (canadienne-française) sans mettre à jour les rapports de pouvoir et la situation inégale qui existent entre les deux? L'une constitue la société d'accueil, et par le biais de son gouvernement, démocratiquement élu, établit les règles du vivre ensemble et détermine les règles du jeu (de l'immigration, aux lois sur la langue etc.), même si elle doit s'accommoder de rapports inégaux en son sein, avec les Premières Nations, les Anglo-Québécois et Canadiens...; tandis que l'autre doit s'accommoder, s'intégrer et faire au mieux avec les éléments dont elle dispose (usage de plusieurs langues, valorisation accrue de l'éducation supérieure, etc.).

C'est en commençant par distinguer les différences intrinsèques à chaque groupe (rapports de classe entre riches et pauvres, entre hommes et femmes, 
etc.), et par montrer comment le Québec s'est transformé au cours du siècle dernier que l'on pourra véritablement faire éclater les stéréotypes. De la nation canadienne-française d'abord constituée et colonisée par les Français et ensuite dominée par les Anglais, ou par le Fédéral, à la société québécoise contemporaine, de multiples rapports de domination (ethnique, religieuse, sexuelle, etc.) se sont transformés en rapports plus égalitaires et civiques, sans qu'ils soient jamais égaux. Le dialogue avec les autres, dont les Juifs, pourrait devenir fructueux si cette réalité des rapports de pouvoir était clairement assumée de part et d'autre au sein de cette société plurielle proposée par l'éminent historien du Québec ${ }^{1}$.

C'est ce que montrent quelques-uns des textes de ce recueil, qui abordent ces rapports sous un angle plus circonscrit, comme celui de Sylvie Taschereau sur le commerce à Montréal entre 1900 et 1945. On y découvre l'épaisseur et la complexité des relations entre Juifs et non Juifs à partir de l'étude de l'espace ouvert par le commerce de détail et les relations d'affaires. Ces rapports impliquent une transformation profonde des représentations de soi et des autres qu'il s'agit de mieux comprendre.

Le texte de Jack Jedwab a le mérite de situer l'analyse de la communauté juive du Québec dans son contexte nord-américain, où elle apparaît comme étant la plus attachée à son identité juive. Est-ce le fruit d'un plus grand activisme communautaire, ou est-ce lié aux conditions socio-économiques particulières au Québec? Pour répondre à ces questions, Jedwab a recours à une étude sociologique de la communauté à partir des données de diverses enquêtes depuis 1972 : une proportion importante, les Juifs de Montréal, s'identifie davantage en termes d'appartenance religieuse qu'ethnique (recensement de 1991), tandis qu'au Québec prédomine le rapport à une seule origine ethnique (p. 63). Le rapport à la langue a profondément été modifié, avec le déclin rapide du yiddish (qui était la première langue parlée par $99 \%$ de la communauté en 1931) au profit de l'anglais majoritaire en 1991 (30\% des autres étant allophones). La distance sociale mesurée par le taux d'intermariage (taux d'exogamie parmi les plus bas d'Amérique du Nord) et par les relations sociales ( $85 \%$ des Juifs disent ne fréquenter que des Juifs, en 1996) révèle l'existence d'une forte ségrégation sociale. Ce qui permettrait de dire que le haut taux de rétention de l'identité juive au Québec

1. Au tout début de son texte, Gérard Bouchard entrevoit bien cette relation «entre les grandes composantes ethniques ou culturelles de cette nation (québécoise), à savoir les Canadiens français, les Autochtones, les Anglo-Québécois et ce qu'il est convenu d'appeler les communautés culturelles" (p. 13), pour élaborer ensuite sur les droits et les devoirs de chacune des communautés, juives et canadiennes-françaises. 
serait plutôt le résultat de facteurs démographiques, sociaux et institutionnels que de l'activisme communautaire. Toutefois, il faut également considérer que l'inconfort que cette communauté ressent au Québec provient également du fait qu’elle est peu représentée dans les instances de décision politique et administratives(pas ou peu de Juifs dans la fonction publique par exemple).

Cette situation paradoxale d'une communauté juive, forte et vivante, mais relativement repliée sur elle-même est présentée par Ignaki Olazabal qui reprend le terme mé-rencontre proposé par Bauman (p. 109). Insistant sur la transformation profonde des valeurs véhiculées par la quatrième génération de Juifs au Québec, il montre l'importance des processus de reconnaissance mutuelle, à l'échelle montréalaise par exemple, comme façon de rapprocher les solitudes (p. 124). Le beau texte de Bernard Dansereau sur le rôle essentiel des travailleurs juifs dans la constitution du mouvement ouvrier et associatif québécois au début du siècle témoigne pourtant du peu de contacts entre les deux groupes.

Au total, des textes solidement ancrés dans la recherche historique récente, ainsi que des textes plus convenus sur les différences de perception internes à la communauté juive (entre anglophones et francophones), ou au sein de la communauté anglophone, avec les détours obligés sur la place du Juif dans la littérature, québécoise et montréalaise. De l'ensemble se dégage néanmoins le sentiment d'avoir affaire à une entreprise politique (il manque les travaux pourtant nombreux sur les Juifs sépharades à Montréal ainsi que les études sur les rapports de genre, très avancées dans les deux groupes). Car l'essentiel de la recherche sur les Canadiens français n'apparaît guère dans cet ouvrage, ce que les directeurs de la collection reconnaissent d'ailleurs aisément.

Alors de quoi s'agit-il au juste? De permettre à la recherche sur les Juifs au Québec d'être connue du public francophone : ce serait déjà un pas de franchi dans la connaissance et reconnaissance mutuelle tant souhaitée par les participants. Il y a plus, toutefois dans cet ouvrage, c'est la volonté de constituer un espace d'échanges publics commun qui permette de débusquer les stéréotypes les plus ancrés, et de faire avancer de quelques pages, un projet national qui n’a pas jusque-là réussi à faire une plus grande place à ses minorités. Et à ce titre, on ne peut que féliciter les organisateurs et directeurs de cet ouvrage d'avoir tenté l'aventure. À quand la suite? 